\title{
Desafios à saúde da criança na Atenção Primária: revisão de literatura integrativa
}

\author{
Challenges to children's health in Primary Care: integrative literature review \\ Desafíos para la salud de los niños en la Atención Primaria: revisión de la literatura integrativa
}

Recebido: 14/01/2021 | Revisado: 18/01/2021 | Aceito: 20/01/2021 | Publicado: 25/01/2021

Laís Roncato de Carvalho Alves
ORCID: https://orcid.org/0000-0002-8387-5536
Universidade José do Rosário Vellano, Brasil
E-mail: laisroncato@ gmail.com
Rodolfo Alvarenga Bastos
ORCID: https://orcid.org/0000-0001-6257-1608
Universidade José do Rosário Vellano, Brasil
E-mail: rodolfobastos@gmail.com
Talissa Tavares Vilela
ORCID: https://orcid.org/0000-0002-8956-9257
Universidade José do Rosário Vellano, Brasil
E-mail: talissavilela@ gmail.com
Ciderleia Castro de Lima
ORCID: https://orcid.org/0000-0002-7737-3935
Universidade José do Rosário Vellano, Brasil
E-mail: ciderleiacastro@yahoo.com.br

\section{Resumo}

Introdução: As políticas de saúde no âmbito materno infantil têm ganhado êxito ao longo dos anos. A população infantil representa $26 \%$ da população mundial, enquanto no Brasil, essa população atinge $21,10 \%$. Vale destacar o papel dos Agentes Comunitários de Saúde na melhoria dos resultados de saúde materno infantil, tanto nos países de baixa e média renda quanto em comunidades menos favorecidas Objetivo: Identificar, na literatura, as principais dificuldades encontradas por profissionais de saúde na assistência de puericultura, às crianças na atenção primária. Material e métodos: Realizou-se uma revisão de literatura integrativa sobre a puericultura com enfoque na qualidade dos atendimentos realizados na atenção primária. Foram pesquisadas publicações de 2015 a 2019 nas bases Medline, PubMed e SciElo. Priorizando publicações de 2017 a 2019 para análise, elas foram separadas em grupos de A a G segundo seus desenhos de pesquisa e níveis de evidência científica. Resultados: Os achados corroboraram para a análise de como é o atendimento na atenção primária para com o público infantil. De maneira específica, a puericultura ainda carece de profissionais bem capacitados e com vontade de lidar com o público pediátrico. É possível perceber a falta de incentivos públicos e de uma boa gestão nas unidades de saúde primária para que os serviços sejam oferecidos satisfatoriamente. Conclusão: Esforços têm sido concentrados para garantir o melhor atendimento na puericultura nas unidades básicas de saúde. Entretanto, ainda há falta de interesse, falta de comprometimento com o público infantil, gestão pública deficiente e reduzidos incentivos financeiros.

Palavras-chave: Puericultura; Saúde da criança; Atenção Primária.

\begin{abstract}
Introduction: Maternal and child health policies have been successful over the years. The child population represents $26 \%$ of the world population, while in Brazil, this population reaches $21.10 \%$. It is worth highlighting the role of Community Health Agents in improving maternal and child health outcomes, both in low- and middle-income countries and in less favored communities Objective: To identify, in the literature, the main difficulties encountered by health professionals in childcare for children in primary care. Material and methods: An integrative literature review on childcare was conducted with a focus on the quality of care in primary care. Publications were searched from 2015 to 2019 at Medline, PubMed and SciElo databases. Prioritizing publications from 2017 to 2019 for analysis, they were separated into groups a to $\mathrm{G}$ according to their research designs and levels of scientific evidence. Results: The findings corroborated the analysis of how primary care is for children. In a specific way, childcare still lacks well-trained professionals with a desire to deal with the pediatric public. It is possible to notice the lack of public incentives and good management in primary health units so that the services are offered satisfactorily. Conclusion: Efforts have been concentrated to ensure the best care in childcare in basic health units. However, there is still a lack of interest, lack of commitment to children, poor public management and reduced financial incentives.
\end{abstract}

Keywords: Childcare; Child health; Primary Care. 


\begin{abstract}
Resumen
Introducción: Las políticas de salud maternoinfantil han tenido éxito a lo largo de los años. La población infantil representa el 26\% de la población mundial, mientras que en Brasil, esta población alcanza el 21,10\%. Cabe destacar el papel de los agentes comunitarios de salud en la mejora de los resultados de la salud maternoinfantil, tanto en los países de ingresos bajos como en los de ingresos medios y en las comunidades menos favorecidas Objetivo: Identificar, en la literatura, las principales dificultades que enfrentan los profesionales de la salud en el cuidado de los niños en la atención primaria. Material y métodos: Se llevó a cabo una revisión de la literatura integradora sobre el cuidado de los niños, centrándose en la calidad de la atención primaria. Las publicaciones se buscaron de 2015 a 2019 en las bases de datos Medline, PubMed y SciElo. Priorizando las publicaciones de 2017 a 2019 para su análisis, se separaron en grupos a a $\mathrm{G}$ de acuerdo con sus diseños de investigación y niveles de evidencia científica. Resultados: Los hallazgos corroboraron el análisis de cómo la atención primaria es para los niños. De una manera específica, el cuidado de niños todavía carece de profesionales bien capacitados con el deseo de tratar con el público pediátrico. Es posible notar la falta de incentivos públicos y una buena gestión en las unidades de salud primaria para que los servicios se ofrezcan satisfactoriamente. Conclusión: Se han concentrado esfuerzos para garantizar la mejor atención en el cuidado de niños en unidades de salud básica. Sin embargo, sigue habiendo falta de interés, falta de compromiso con los niños, mala gestión pública y reducción de los incentivos financieros.
\end{abstract}

Palabras clave: Cuidado de niños; Salud infantil; Atención Primaria.

\title{
1. Introdução
}

As políticas de saúde no âmbito materno infantil têm ganhado êxito ao longo dos anos. Diversos mecanismos de intervenção foram desenvolvidos mundialmente, entre eles propostas que visam cuidados primários de saúde (WHO, 1978). Desta maneira, diversas nações têm adotado ações na atenção básica como estratégias de cuidados pediátricos (Bonne et al., 2017, Melo \& Lima, 2018, Pinto-Junior et al., 2018ª Fernández et al., 2019). Estima-se que o número de crianças entre 0 e 14 anos no mundo seja de 2 bilhões, o que representa 26\% da população mundial (UNFPA, 2019), enquanto no Brasil, essa população atinge $21,10 \%$ (IBGE, 2019).

E diante desse cenário ações abrangendo a promoção da saúde comunitária e a provisão de serviços de saúde conquistaram bons indicadores, sendo o principal a redução da mortalidade infantil (Bonne et al., 2017). Vale destacar o papel dos Agentes Comunitários de Saúde na melhoria dos resultados de saúde materna e infantil, particularmente em países de baixa e média renda e em comunidades desfavorecidas (Peacock-Chambers, Ivy \& Bair-Merritt, 2017, Rotheram-Borus et al., 2017, Baqui et al., 2018).

No contexto atual de boas práticas em saúde na pediatria, sobretudo na assistência baseada em evidências, a revisão integrativa se faz necessária para sintetizar conhecimento e incorporar os resultados no exercício profissional (Soares et al., 2014). O presente trabalho teve como objetivo identificar as principais dificuldades encontradas por profissionais de saúde na assistência de puericultura, às crianças, na atenção primária.

\section{Metodologia}

Trata-se de uma revisão de literatura integrativa de abordagem qualitativa na qual é importante a interpretação por parte do pesquisador com suas opiniões sobre o fenômeno em estudo ( Pereira, Shitsuka, Parreira \& Shitsuka, 2018) sendo um estudo com coleta de dados realizado a partir de fontes secundárias, por meio de levantamentos bibliográficos contemplando 6 etapas: seleção por palavras-chave, seleção por ano de publicação, leitura, análise, nova seleção e escrita . Para o levantamento de artigos de literatura, utilizou-se como base de dados: SciElo, Medical Literature Analysis and Retrieval Sistem on-line (Medline) e Pubmed. Foram utilizados, para busca dos artigos, os seguintes descritores: "Puericultura" and "Saúde da criança" and "Atenção primária".

O critério para realizar a pesquisa teve início com a seleção de artigos que contemplavam as palavras chaves do trabalho, sendo encontrados 2908. Depois, foi estabelecido o filtro com publicações que foram compreendidas entre os anos de 2015 a 2019. Após essa seleção, foram selecionados os trabalhos que atendessem a pergunta guia da revisão: "Quais são as dificuldades 
encontradas no atendimento às crianças na atenção básica?”. Os trabalhos que não atenderam à pergunta foram excluídos do estudo, e os trabalhos selecionados passaram por mais um filtro com publicações compreendidas entre os anos de 2017 a 2019. Assim, foram selecionados 13 artigos.

O propósito geral de uma revisão de literatura é reunir conhecimentos sobre um tópico, ajudando nas fundações de um estudo significativo para a área médica. Para isso, o estudo é desenvolvido por fases.

Para a primeira fase desse estudo buscou por artigos que discutiam sobre o atendimento infantil nas unidades básicas de saúde e como esses atendimentos eram realizados, e se eram realizados de maneira satisfatória. Com isso, buscou selecionar artigos mais recentes (últimos três anos) a fim de analisar uma realidade mais atual do cenário da puericultura. Para a segunda fase construiu-se uma tabela para o agrupamento das informações necessárias à análise, como: categoria do artigo, autoria/ano de publicação, título, tipo de estudo, resultados e considerações.

Em seguida, os trabalhos foram devidamente separados por categorias, onde foram incluídos nas categorias de "A" a "G", para uma melhor organização dos resultados coletados. Sendo assim, a categoria A inclui trabalho de estudo transversal, a B trabalhos de estudos de intervenção, a C trabalho de estudos exploratórios, a D os estudos ecológicos, a E estudos de coorte com perspectiva diagnóstica, a $\mathrm{F}$ estudos randomizados e a $\mathrm{G}$ revisão de literatura.

A coleta de dados e o agrupamento dos artigos compreendeu os meses de março a junho de 2019, e a redação dos resultados compreendeu os meses de julho a novembro de 2019.

Com os dados devidamente agrupados, procedeu-se com a terceira fase. Nessa foi utilizada a análise qualitativa dos estudos, observando as ações dos agentes de saúde, como os atendimentos de puericultura são realizados, a falta de estrutura, incentivo e manejo das unidades básicas para atender ao público infantil, além da falta de incentivo público (renda governamental) para garantir um atendimento adequado desde a primeira infância de maneira gratuita e de qualidade.

Em relação aos aspectos éticos, para esse tipo de estudo, dispensa- se a apreciação pelo comitê de ética em pesquisa.

\section{Resultados e Discussão}

Os dados obtidos por esse estudo foram agrupados em categorias de A a G para uma melhor análise o que possibilitou identificar o atual cenário do atendimento por profissionais na atenção primária voltado para a puericultura e para a pediatria, resultados sintetizados no Quadro 1 desse estudo.

Nos estudos transversais (categoria A) é utilizada a estratégia de exposição e desfecho sobre um determinado tema. Sendo assim, no estudo realizado com o auxílio de algumas mães, foi possível detectar a satisfação delas com o atendimento proporcionado às suas crianças pelos profissionais de saúde nas unidades de Atenção Primária à Saúde (APS) no estado da Paraíba, onde, foram sim, encontrados resultados satisfatórios (Perdaza \& Santos, 2018). Entretanto, os resultados insatisfatórios prevaleceram ao ponto de levantar o questionamento sobre o porquê da permanência de um atendimento ainda insatisfatório para com o público infantil. Na tentativa de encontrar uma explicação para tal atendimento, outro autor mostra em seu trabalho uma falta de efetivação dos avanços preconizados pelas políticas de atenção à saúde da criança que acabam prejudicando no avanço de um atendimento que satisfaça aos princípios do SUS, principalmente, o da longitudinalidade (Sanini et al., 2018).

Não obstante, ainda é possível se deparar com a falta de esforços das gestões locais e de organização dos serviços de capacitação dos profissionais que trabalham nas unidades de atenção primária. Foi então, em um estudo clínico não randomizados (categoria B) onde há um grupo intervenção e um grupo controle, porém a designação dos participantes para cada grupo não se dá de forma aleatória, como no Estudo Clínico Randomizado (ECR), mas por conveniência do pesquisador (Thiese, 2014), que um grupo de enfermeiros, auxiliares de enfermagem e agentes comunitários de saúde foram separados para realizar um estudo crítico sobre o aconselhamento nutricional na infância (Palombo et al., 2018). Este delineamento não consegue controlar outros fatores que podem ter ocorrido concomitantes à intervenção implantada, e que podem ter contribuído para a mudança no desfecho 
(Nedel \& Silveira, 2016). E por mais que os profissionais possam ter buscado meios de atualização, o efeito de levar realizar capacitação aos profissionais de saúde que atuam na atenção primária, reflete em uma maior habilidade desses profissionais em atuarem na promoção e prevenção de saúde.

Ainda na tentativa de atualização dos profissionais, foi possível observar que a não capacitação dos profissionais os impedem de atuarem em certos momentos dentro das unidades de atenção primária (Teixeira, Couto \& Delgado, 2017). Os profissionais de saúde identificam o problema, contudo, não são capazes de atuar na resolução dos mesmos. Nesse sentido, o estudo (categoria C) de cunho exploratório mostrou que uma das barreiras para essa não competência para resolução se deve à má articulação entre as redes de saúde. Sendo assim, muitos cuidados à saúde da criança ficam defasados por barreiras existentes no SUS.

Habilitar-se a uma função, ainda é uma realidade pouco vista na atenção primária, por falta de incentivos financeiros, por falta de vontade dos profissionais e até mesmo por falta de infraestrutura. Sendo assim, os gastos com o público infantil no âmbito hospitalar têm sido cada vez mais elevados. Basta observar as unidades básicas de saúde para perceber o déficit de materiais, de mão de obra e de incentivos para realização de procedimentos básicos. Com isso, os gastos com internações pediátricas ainda são consideravelmente altos. No estudo realizado na categoria $\mathrm{D}$, foi possível analisar a necessidade de reforçar as ações no âmbito da atenção primária à Saúde da Família, e o fortalecimento de políticas públicas para enfrentar os problemas sociais, objetivando reduzir cada vez mais a ocorrência das internações e de seus gastos (Pinto-Junior et al., 2018b).

Corroboram com esses achados, o apresentado no estudo da categoria E (estudo de coorte com perspectiva de diagnóstico) em que o autor mostrou a incidência de doenças que acometem crianças em tratamento oncológico (De Fine Licht et al., 2017) com possibilidade de se aumentar a incidência de hospitalizações, tendo em vista que, a assistência qualificada na atenção primária contribui para o não aumento de recidiva (Cunha \& Giovanella, 2011). No Brasil, é sabido que os hospitais são ambientes extremamente contaminados e que não é o local ideal para recuperação. Em se tratando de pacientes com o sistema imunológico extremamente debilitado, se recuperar e precisar se internar várias vezes, acaba facilitando a contaminação das crianças e o surgimento de novas doenças. Com isso é possível observar que se a atenção primária fosse melhor equipada, contasse com profissionais bem capacitados e com ambientes devidamente preparados para atender os pacientes recém liberados do hospital, essa incidência de doenças pós tratamento oncológico e de recidivas seriam menores.

Ao analisar outro grupo de estudos, como os ensaios clínicos randomizados - categoria $\mathrm{F}$ (os ensaios clínicos são estudos onde um grupo de interesse em que se faz uso de uma terapia ou exposição é acompanhado comparando-se com um grupo controle), mães gestantes, puérperas e recém-nascidos foram acompanhados de perto pelos agentes de saúde, onde parte dos agentes atuaram de maneira mais simples e outros de maneira mais avançada. Os que atuaram de maneira mais simples, passaram por treinamento orientado para a ação sobre conhecimentos, atitudes e práticas (CAP) em relação à saúde materna e infantil. No final do estudo foi possível perceber que os agentes comunitários de saúde adquiriram as capacidades necessárias para a abordagem das gestantes e dos recém-nascidos durante as visitas domiciliares. Já os que atuaram de forma mais avançada passaram por treinamento em reanimação básica de recém-nascidos e em reconhecimento e tratamento de suspeitas de infecções respiratórias neonatais (Soofi et al., 2017). Foi possível observar a redução das mortes neonatais simplesmente devido a uma melhor instrução que foi repassada dos agentes de saúde às mães e até mesmo por melhor atuação desses profissionais de saúde, tanto nas unidades de atendimento quanto nos atendimentos domiciliares.

Assim, o último estudo da amostra trouxe a importância do Programa de Educação em Saúde (EPS) no momento de definir ser ou não necessária as consultas nos primeiros seis meses de vida. Basta analisar o cenário brasileiro para notar a falta de conhecimento das gestantes e das puérperas por deficiência de um pré-natal bem feito e bem orientado (Lima et al., 2018). Se em todos os estados e municípios os agentes de saúde recebessem os treinamentos básicos e avançados, bem como passassem pelo EPS, as comunidades ficariam bem mais assistidas, os números de mortes seriam reduzidos, as unidades básicas de saúdes 
não seriam superlotadas e os gastos onerosos com hospitalizações seriam reduzidos consideravelmente.

Quadro I - Agrupamento das publicações segundo a análise dos desenhos de pesquisa. (N-13).

\begin{tabular}{|c|c|c|c|}
\hline CATEGORIAS & \multicolumn{3}{|c|}{$\mathbf{A}$} \\
\hline $\begin{array}{l}\text { AUTORIA/ANO } \\
\text { DE } \\
\text { PUBLICAÇÃO }\end{array}$ & Pedraza, D.F., \& Santos, I.S. $/ 2018$ & Sanine et al. $/ 2018$ & Santos / 2018 \\
\hline TÍTULO & $\begin{array}{c}\text { Percepção materna do cuidado à } \\
\text { saúde da criança na estratégia } \\
\text { saúde da família de dois da } \\
\text { Paraíba, Brasil }\end{array}$ & $\begin{array}{l}\text { Do preconizado à prática: oito anos } \\
\text { de desafios para a saúde da criança } \\
\text { em serviços de atenção primária no } \\
\text { interior de São Paulo, Brasil }\end{array}$ & $\begin{array}{l}\text { Presença e extensão dos } \\
\text { atributos de atenção } \\
\text { primária à saúde da criança } \\
\text { em distintos modelos de } \\
\text { cuidado }\end{array}$ \\
\hline $\begin{array}{l}\text { DESENHO DE } \\
\text { ESTUDO }\end{array}$ & $\begin{array}{l}\text { Prospectivo não randomizado } \\
\text { (estudo transversal) }\end{array}$ & $\begin{array}{l}\text { Prospectivo não randomizado } \\
\text { (estudo transversal) }\end{array}$ & $\begin{array}{l}\text { Prospectivo não } \\
\text { randomizado } \quad \text { (estudo } \\
\text { transversal) }\end{array}$ \\
\hline RESULTADOS & $\begin{array}{l}\text { Nos dois municípios estudados, } \\
\text { foram obtidos resultados } \\
\text { satisfatórios e insatisfatórios } \\
\text { quanto à análise dos cuidados } \\
\text { prestados as crianças menores de } \\
\text { cinco anos pelos agentes da ESF }\end{array}$ & $\begin{array}{l}\text { Conclui-se que os avanços } \\
\text { preconizados pelas políticas de } \\
\text { atenção à saúde da criança não têm } \\
\text { se efetivado plenamente na } \\
\text { organização da oferta da atenção } \\
\text { integral, ainda que temas relevantes } \\
\text { como obesidade infantil e violência } \\
\text { sejam abordados em um pequeno } \\
\text { número de serviços }\end{array}$ & $\begin{array}{l}\text { Os resultados permitem } \\
\text { verificar a percepção de } \\
1.484 \text { familiares e/ou } \\
\text { cuidadores de crianças } \\
\text { menores de } 10 \text { anos sobre a } \\
\text { qualidade da atenção } \\
\text { ofertada pelos distintos } \\
\text { modelos de APS }\end{array}$ \\
\hline $\begin{array}{l}\text { CONSIDERA } \\
\text { ÇÕES }\end{array}$ & $\begin{array}{l}\text { Melhorar a qualidade da APS em } \\
\text { termos de acessibilidade, } \\
\text { longitudinalidade e integralidade }\end{array}$ & $\begin{array}{l}\text { Pesquisas ecológicas evidenciam } \\
\text { melhorias nos níveis de saúde } \\
\text { infantil, incluindo a redução da } \\
\text { mortalidade infantil, mas apontam } \\
\text { ao mesmo tempo uma piora dos } \\
\text { serviços da APS }\end{array}$ & $\begin{array}{l}\text { As unidades de UBS } \\
\text { mista, tem se mostrado } \\
\text { mais eficientes do que os } \\
\text { modelos de atendimento } \\
\text { tradicionais }\end{array}$ \\
\hline
\end{tabular}

\begin{tabular}{|c|c|c|c|}
\hline $\mathbf{B}$ & $\mathbf{C}$ & & D \\
\hline Palombo et al. /2018 & Teixeira et al. $/ 2017$ & Junior et al. $/ 2018$ & Birnie et al./ 2017 \\
\hline $\begin{array}{c}\text { Treinamento em } \\
\text { aconselhamento } \\
\text { nutricional: avaliação } \\
\text { do conhecimento e } \\
\text { aplicabilidade em } \\
\text { cuidados de saúde da } \\
\text { criança }\end{array}$ & $\begin{array}{c}\text { Atenção básica e } \\
\text { cuidado colaborativo } \\
\text { na atenção psicossocial } \\
\text { de crianças e } \\
\text { adolescentes: } \\
\text { facilitadores e barreiras }\end{array}$ & $\begin{array}{c}\text { Tendência dos gastos e das } \\
\text { internações por condições } \\
\text { sensíveis à Atenção Primária em } \\
\text { menores de cinco anos na Bahia, } \\
\text { Brasil }\end{array}$ & $\begin{array}{l}\text { Comparação do diagnóstico } \\
\text { microbiológico da infecção do trato } \\
\text { urinário em crianças pequenas por } \\
\text { laboratórios de serviços de saúde de } \\
\text { rotina e um laboratório de pesquisa: } \\
\text { Estudo de coorte diagnóstico }\end{array}$ \\
\hline $\begin{array}{c}\text { Prospectivo não } \\
\text { randomizado (estudo } \\
\text { de intervenção) }\end{array}$ & $\begin{array}{c}\text { Prospectivo não } \\
\text { randomizado (estudo } \\
\text { exploratório) }\end{array}$ & Retrospectivo (estudo ecológico) & $\begin{array}{l}\text { Prospectivo randomizado (estudo de } \\
\text { coorte) }\end{array}$ \\
\hline
\end{tabular}




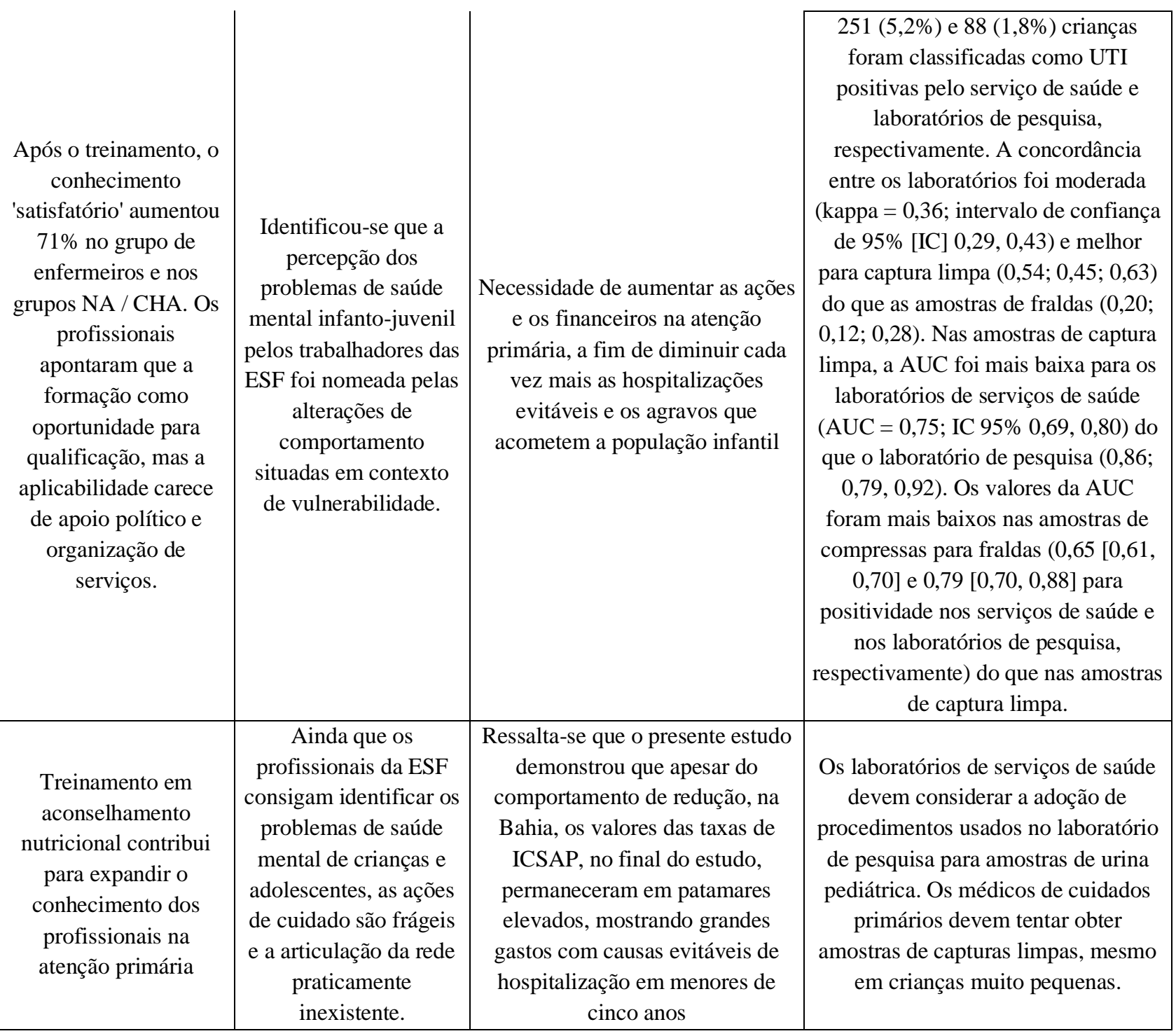

\begin{tabular}{|c|c|c|}
\hline $\mathbf{E}$ & \multicolumn{2}{|c|}{$\mathbf{F}$} \\
\hline de Fine Licht S et al. / 2017 & Fernández et al / 2019 & Boone, Peter et al /2017 \\
\hline $\begin{array}{l}\text { Long-term inpatient disease burden in the } \\
\text { Adult Life after Childhood Cancer in } \\
\text { Scandinavia (ALiCCS) study: A cohort study } \\
\text { of 21,297 childhood cancer survivors }\end{array}$ & $\begin{array}{c}\text { Intervención Educativa En Atención } \\
\text { Primaria Para Reducir Y Mejorar La } \\
\text { Adecuación De Las Consultas } \\
\text { Pediátricas }\end{array}$ & $\begin{array}{l}\text { Community health promotion } \\
\text { and medical provision for } \\
\text { neonatal health-CHAMPION } \\
\text { cluster randomised trial in } \\
\text { Nagarkurnool district, Telangana } \\
\text { (formerly Andhra Pradesh), India }\end{array}$ \\
\hline Prospectivo randomizado (estudo de coorte) & $\begin{array}{c}\text { Prospectivo randomizado (estudo } \\
\text { experimental) }\end{array}$ & Prospectivo randomizado \\
\hline
\end{tabular}


Sobreviventes de todos os tipos de câncer infantil estavam com risco aumentado e persistente de hospitalização subsequente, sendo os maiores riscos de sobreviventes de neuroblastoma
Foram analisadas 191 gestantes (101 controles e 90 intervenções). As mulheres grávidas pela primeira vez $(62,3 \%$ de intervenção versus $54,1 \%$ de controle) com o ensino médio treinado $(59,1 \%$ de intervenção versus $44,2 \%$ de controle) foram mais participativas. $\mathrm{O}$ número de consultas pelos motivos estudados foi reduzido (3,5 no grupo controle vs 2,0 no grupo intervenção), e o nível de adequação melhorou em 16,3\% (RR: 1,62; IC95\%: 1,26- 2,07, p $<0,05)$.

Ressalta a diminuição do número de consultas e ao aumento de sua adequação nos levam a recomendar claramente o estabelecimento de entrevistas pré-natais que abordem os problemas de saúde mais comuns em crianças, no contexto da Atenção Básica.
Dos nascidos vivos, $343(6,9 \%)$ no grupo controle e $303(5,2 \%)$ no grupo de intervenção morreram nos primeiros 28 dias de vida, sugerindo que houve 92 mortes a menos como resultado da intervenção. Também encontrou evidências de melhores conhecimentos sobre saúde e práticas de saúde, incluindo o uso de serviços de saúde no grupo de intervenção, em comparação com o grupo de controle.

Os resultados da pesquisa significam que intervenções como as do estudo CHAMPION podem ser fortemente justificadas em grande parte da Índia rural adiar hospitalizações por prevenção, detecção precoce e tratamentos adequados.

\begin{tabular}{|c|c|c|}
\hline \multicolumn{2}{|l|}{$\mathbf{F}$} & $\mathbf{G}$ \\
\hline Soofi, Sajid et al / 2017 & Lima, et al / 2018 & Patel / 2018 \\
\hline $\begin{array}{l}\text { Effect of provision of home-based curative } \\
\text { health services by public sector health-care } \\
\text { providers on neonatal survival: a community- } \\
\text { based cluster-randomised trial in rural } \\
\text { Pakistan }\end{array}$ & $\begin{array}{l}\text { Effectiveness of an action-oriented } \\
\text { educational intervention in ensuring long } \\
\text { term improvement of knowledge, } \\
\text { attitudes and practices of community } \\
\text { health workers in maternal and infant } \\
\text { health: a randomized controlled study }\end{array}$ & $\begin{array}{c}\text { Structural, institutional and } \\
\text { organizational factors associated } \\
\text { with successful pay for } \\
\text { performance programmes in } \\
\text { improving quality of maternal } \\
\text { and child health care in low and } \\
\text { middle income countries: a } \\
\text { systematic literature review }\end{array}$ \\
\hline $\begin{array}{c}\text { A taxa de mortalidade neonatal foi de } 42 \\
\text { mortes por } 1.000 \text { nascidos vivos em grupos de } \\
\text { intervenção em comparação com } 55 \text { por } 1000 \\
\text { no grupo controle (risk ratio } 0,80, \text { IC } 95 \% \\
0,68-0,093 ; p=0,005) .\end{array}$ & $\begin{array}{c}\text { Cinquenta e nove ACS completaram } \\
\text { todas as avaliações da CAP ( } 31 \text { na } \\
\text { intervenção e } 28 \text { no grupo controle). As } \\
\text { características basais foram semelhantes } \\
\text { nos dois grupos. Ao } 1 \text { ano de treinamento, } \\
\text { o grupo de intervenção teve maior } \\
\text { pontuação CAP global }(120,65 \text { vs } 108,19, \\
\text { p <0,001), bem como conhecimento } \\
\text { (47,45 vs } 40,54, p<0,001) \text {, prática ( } 53,45 \\
\text { vs } 49,11, p<0,001) \text { e escores de atitudes } \\
(19,74 \text { vs. } 18,81, p=0,047) \text { do que o } \\
\text { grupo controle. Além disso, com } 1 \text { ano de } \\
\text { treinamento, o grupo de intervenção } \\
\text { manteve melhorias significativas no } \\
\text { escore total da CAP }(120,65 \text { vs } 106,55, p \\
<0,001), \text { bem como no conhecimento } \\
(45,45 \text { vs } 42,13, p<0,001) \text { e prática } \\
(53,45 \text { vs. } 45,29, p<0,001) \text { pontuações } \\
\text { em relação à linha de base. }\end{array}$ & $\begin{array}{l}\text { A maioria dos estudos encontrou } \\
\text { um impacto positivo na qualidade } \\
\text { dos escores de cuidados e pelo } \\
\text { menos um estudo mostrou } \\
\text { reduções significativas nos } \\
\text { desfechos de mortalidade em } \\
\text { recém-nascidos }\end{array}$ \\
\hline
\end{tabular}




\begin{tabular}{|c|c|c|}
\hline Prospectivo randomizado & $\begin{array}{l}\text { Prospectivo randomizado (estudo de } \\
\text { intervenção) }\end{array}$ & $\begin{array}{c}\text { Retrospectivo (revisão de } \\
\text { literatura) }\end{array}$ \\
\hline $\begin{array}{l}\text { A redução da mortalidade neonatal nos grupos } \\
\text { de intervenção ocorreu em um cenário de } \\
\text { melhorias nas práticas domiciliares para os } \\
\text { cuidados maternos e neonatais. }\end{array}$ & $\begin{array}{l}\text { Um curso de treinamento interativo de } \\
\text { quatro dias sobre visitas domiciliares } \\
\text { orientadas para a ação a mulheres } \\
\text { grávidas e bebês produziu uma melhoria } \\
\text { sustentada na CAP do ACS e pode } \\
\text { representar um modelo para garantir a } \\
\text { retenção das competências adquiridas }\end{array}$ & $\begin{array}{l}\text { Os fatores estruturais associados } \\
\text { ao provável sucesso dos } \\
\text { programas de P4P incluem: } \\
\text { aceitação explícita e compreensão } \\
\text { pelos profissionais de saúde; } \\
\text { limitando o número de } \\
\text { indicadores medidos com } \\
\text { insumos de trabalhadores de } \\
\text { saúde. Fatores organizacionais } \\
\text { incluíram pagamentos de } \\
\text { incentivo suficientes. }\end{array}$ \\
\hline
\end{tabular}

Fonte: Alves, et al. (2021).

Assim, pelos achados descritos e apresentados no Quadro 1 observa-se dificuldades na assistência à atenção primária no âmbito da puericultura, como a deficiência na infraestrutura das unidades de atendimento, falta de incentivos financeiros, pouca organização de serviços de capacitação, e falta de profissionais da saúde bem preparados para atender ao público infantil. Além das dificuldades estruturais, foi possível perceber falhas estratégicas, como no atendimento longitudinal dos pacientes nas unidades de atenção primária, sendo a longitudinalidade um acompanhamento do paciente por um longo período de tempo (Cunha \& Giovanella, 2011).

A falta de comprometimento com o público infantil abre espaço para possíveis questionamentos: Por que o descaso? A remuneração ainda é precária? Os profissionais estão sendo bem preparados para lidar com o público infantil? Com a falta de capacitação dentro dos serviços de saúde, é possível perceber que açães educativas no âmbito da promoção e prevenção de saúde ficam comprometidas, resultando em um atendimento ineficiente por parte dos profissionais. As ações de educação em saúde se fazem de extrema importância, no sentido de melhorar o atendimento, tanto para o público infantil quanto os profissionais, consequentemente as crianças conseguem ter um atendimento mais direcionado para as suas necessidades, e o profissional conseguem lidar melhor com os seus pacientes desde a abordagem até às recomendações finais. Sendo assim, transpor a barreira da educação em saúde gera resultados positivos para toda a sociedade, gerando melhor prestação de assistência na saúde (Godoy, Guimarães \& Assis, 2014).

$\mathrm{Na}$ tentativa de prestar assistência efetiva, às unidades de atenção primária devem contar com equipes multiprofissionais atuantes, as quais podem ofertar às crianças grupos de atividade física, grupos de teatro e grupos de conversa, além da assistência médica e nutricional de acordo com as idades. Dessa forma, é possível que o atendimento à puericultura seja de fato algo mais difundido nas unidades de saúde, entre as crianças e a sociedade. Nota-se que melhorias já foram alcançadas no âmbito do atendimento infantil, mas ainda há muito a melhorar.

A comunicação entre os diversos serviços de saúde do Sistema Único de Saúde (SUS) carecem de uma relação mais próxima. Com o avanço da tecnologia e a estratégia de informação em rede, juntamente com a falta de tempo da população, é importante que estratégias efetivas sejam pensadas, como a construção de redes ampliadas de atenção à saúde infantil, a qual consegue garantir desde acesso a cuidados qualificados dentro da puericultura. Assim, uma articulação intersetorial potente entre os diversos serviços do SUS pode construir-se como a base de sustentação para o desenvolvimento da colaboração, compartilhamento e corresponsabilidade entre diferentes setores de assistência à infância e adolescência, permitindo a instauração de novos modos de cuidar que ultrapassem a fragmentação e a dispersão (Teixeira, Couto \& Delgado, 2017). 


\section{Conclusão}

Os estudos aqui revisados evidenciaram que existem falhas no planejamento e na assistência à atenção primária no âmbito da puericultura, que vão desde deficiência na infraestrutura das unidades de atendimento, falta de planejamento e incentivos financeiros e profissionais capacitados. Nota-se que recentes melhorias estão sendo realizadas tanto para os profissionais envolvidos no atendimento quanto para o público infantil.

Contudo, há muito o que melhorar, principalmente no quesito investimento, a qual permitirá que as lacunas existentes neste setor sejam supridas. Visto que, se em todos os estados e municípios os agentes de saúde, bem como as unidades de atendimento recebessem suportes básicos e avançados e passassem pelo EPS, o atendimento à comunidade seria mais eficaz e melhor assistido, e em consequência, o índice de mortalidade reduzirá, as unidades básicas de saúdes não seriam superlotadas e os gastos onerosos com hospitalizações seriam reduzidos consideravelmente.

Diante do trabalho nota-se como um desafio buscar compreender as principais dificuldades encontradas na saúde da criança na atenção primária. Além disso, faz-se necessário integrar o conhecimento adquirido nesse campo da ciência para desenvolver as ações de cuidado e melhorias nas redes de atendimento ao público infantil.

\section{Referências}

Baqui, A. H., Ahmed, S., Begum, N., Khanam, R., Mohan, D., Harrison, M., Kabir, A., McKaig, C., Brandes, N., Nortin, M., \& Ahmed, S. (2018). Impact of integrating a postpartum family planning program into a community-based maternal and newborn health program on birth spacing and preterm birth in rural Bangladesh. J Glob Health, 8(2), 020406. 10.7189/jogh.08.020406.

Boone, P., Eble, A., Elboume, D., Frost, C., Jayanty, C., Lakshminarayana, R., Mann, V., Mukherjee, R., Pjaggio, G., \& Reddy, P. (2017). Community healthpromotion and medical provision for neonatal heptanal-CHAMPION clusterrandomised trial in Nagarkurnool district, Telangana (formerly Andhra Pradesh). P Med, 14(7), 1002324.

Cunha, E. M., \& Giovanella, L. (2011). Longitudinalidade/continuidade do cuidado: identificando dimensões e variáveis para a avaliação da Atenção Primária no contexto do sistema público de saúde brasileiro. Ciênc. saúde coletiva, 16(1), 1029-1042.10.1590/S1413-81232011000700036.

De Fine Licht, S., Rugbjerg, K., Gudmundsdottir, T., Bonnesen, T. G., Asdahl, P. H., Holmqvist, A. S., Madanat-Harjuoja, L., Tryggvadottir, L., Wesenberg, F., Hasle, H., \& Winther, J. F. (2017) Long-term inpatient disease burden in the Adult Life after Childhood Cancer in Scandinavia (ALiCCS) study: A cohort study of 21,297 childhood cancer survivors. PLoS Med, 14(5), e1002296. https://doi.org/10.1371/journal.pmed.1002296

Fernández, M. E. V., Almazán, M. A., Sanz, S. G., Villalibre, C. B., González, M. A., \& López, A. R. (2019). Intervención educativa enatención primaria para reducir y mejorar la adecuación de las consultas pediátricas. Rev Esp Salud Pública, e201901003.

Godoy, S. C. B., Guimarães, E. M. P., \& Assis, D. S. S. (2014). Avaliação dos enfermeiros por telenfermagem. Escola Anna Nery Revista de Enfermagem, 18(1), $148-155$.

IBGE - Instituto Brasileiro de Geografia e Estatística. (2019). Projeções e estimativas da população do Brasil e das Unidades da Federação. https://www.ibge.gov.br/apps/populacao/projecao/.

Lima, T. R., Maia, P. F. C. M. D., Valente, E. P., Vezzini, F., \& Tamburlini, G. (2018). Effectiveness of anaction-oriented educational intervention in ensuring long term improvement of knowledge, attitudes and practices of community health workers in maternal and infant health: a rand omized controlled study. $B M C$ Medical Education, 18(1)224. 10.1186/s12909-018-1332-x.

Nedel, W. L., \& Silveira, F. (2016). Os diferentes delineamentos de pesquisa e suas particularidades na terapia intensiva. Rev. Bras. Ter. Intensiva, 28(3), 256260. 10.5935/0103-507X.20160050.

Palombo, C. N. T., Fujimori, E., Toriyama, A. T. M., \& Duarte, L. S. (2018). Training in nutritional counseling: knowledge assessment and applicability in child's healthcare. Rev. Bras. Saude Mater. Infant., 18(1), 67-74. 10.1590/1806-93042018000100003.

Peacock-Chambers, E., Ivy, K., \& Bair-Merritt, M. (2017). Primary Care Interventions for Early Childhood Development: A Systematic Review. Pediatrics, 140(6), e20171661. doi: 10.1542.

Pedraza D.F., \& Santos I.S. (2018). Percepção materna do cuidado à saúde da criança na estratégia saúde da família de dois municípios da Paraíba, Brasil. Rev. Gerenc. Polit. Salud, Bogotá, 17(34), 119-129. 10.11144/javeriana.rgps 17-34.pmcs.

Pereira, A. S., Shitsuka, D. M., Parreira, F. J. \& Shitsuka, R. (2018). Metodologia da pesquisa científica. Ed. UAB/NTE/UFSM.

Pinto Junior, E., Pereira, A. R., Medina, M. G., \& Silva, M. G. C. (2018a). Efeito da Estratégia Saúde da Família nas internações por condições sensíveis à atenção primária em menores de um ano na Bahia, Brasil. Cad. Saúde Pública, 34(2), e00133816. 
Research, Society and Development, v. 10, n. 1, e47610111990, 2021

(CC BY 4.0) | ISSN 2525-3409 | DOI: http://dx.doi.org/10.33448/rsd-v10i1.11990

Pinto Junior, E. P., Costa, L. Q., Oliveira, S. M. A., Medina, M. G., Aquino, R., \& Silva, M. G. C. (2018b). Tendência dos gastos e das internações por condições sensíveis à Atenção Primária em menores de cinco anos na Bahia, Brasil. Ciênc. saúde coletiva, 23(12), 4331-4338. 10.1590/1413-812320182312.32122016.

Rotheram-Borus, M. J., Le Roux, K., Le Roux, I. M., Christodoulou, J., Laurenzi, C., Mbewu, N., \& Tomlinson, M. (2017). To evaluate if increased supervision and support of South African Government health workers' home visits improves maternal and child outcomes: study protocol for a randomized control trial. Trials, 18(1), 368. 10.1186/s13063-017-2074-5.

Sanine, P. R., Zarili, T. F. T., Nunes, L. O., Dias, A., \& Castanheira, E. R. L. (2018). Do preconizado à prática: oito anos de desafios para a saúde da criança em serviços de atenção primária no interior de São Paulo, Brasil. Cad. Saúde Pública, 34(6), e00094417. 10.1590/0102-311x00094417.

Soares, C. B., Hoga, L. A. K., Peduzzi, M. S., Carine, Y. T., \& Silva, D. R. A. D. (2014). Revisão integrativa: conceitos e métodos utilizados na enfermagem. Revista da Escola de Enfermagem da USP, 48(2), 335-345. 10.1590/S0080-6234201400002000020.

Soofi, S., Cousens, S., Turab, A., Wasan, Y., Mohammed, S., Ariff, S., Bhatti, Z., Ahmed, I., Wall, S., \& Bhutta, Z. A. (2017). Effect of provision of homebased curative health services by public sector health-care providers on neonatal survival: a community-based cluster-randomised trial in rural Pakistan. Lancet Glob Health, 5(8), 796-e806. 10.1016/S2214-109X(17)30248-6.

Teixeira, M. R., Couto, M. C. V., Delgado, P. G. G. (2017). Atenção básica e cuidado colaborativo na atenção psicossocial de crianças e adolescentes: facilitadores e barreiras. Ciênc. Saúde Coletiva, 22(6), 1933-1942. 10.1590/1413-81232017226.06892016.

Thiese, M. S. (2014). Observational and interventional study design types; an overview. Biochem Med., 24(2), 199-210. 10.11613/BM.2014.022.

UNFPA - United Nations Population Fund. (2019). World Population Dashboard. https://www.unfpa.org/es/data/world-population-dashboard

WHO - World Health Organization. (1978). Declaration of Alma-Ata: International Conference on Primary Health Care. https://www.euro.who.int/en/publications/policy-documents/declaration-of-alma-ata,-1978 